DOI: $10.21892 / 978-958-5547-66-7.2$

\title{
2. Justicia normal neoliberal en Salud: un análisis crítico de la sentencia T-760/08 de la Corte Constitucional ${ }^{1}$
}

Daniel Alzate Mora²

\section{Introducción}

A inicio de los años noventa, el gobierno de César Gaviria reformó en Colombia el sistema de salud para superar las falencias del anterior Sistema Nacional de Salud. Esta reforma quedó consignada en la Ley 100 de 1993, en consonancia con la Constitución Política de 1991, instaurando un esquema sustentado en el aseguramiento individual y privado, con estratificaciones en cuanto a los servicios prestados según la capacidad de pago, con la participación del Estado y el mercado (Uribe, 2009).

La puesta en marcha del nuevo sistema de salud ha estado acompañada por un mecanismo ajeno al sistema: la acción de tutela (Uribe, 2009). Este mecanismo, creado por la Constituyente de 1991, es una acción judicial de carácter subsidiario y residual para el control constitucional de las autoridades públicas y excepcionalmente, de los particulares (Botero, 2006). Según la Defensoría del Pueblo, para el año 2008 el incremento del número de acciones de tutela en salud fue del 300\% en comparación con las tutelas desde 1999, año a partir del cual dicha entidad tiene registros ${ }^{3}$.

El uso masivo y constante de la tutela para resolver los conflictos con el sistema de salud y sus consecuencias asociadas, entre ellas la congestión

1 Una versión preliminar de este trabajo investigativo fue divulgada con anterioridad en: Alzate Mora, D. (2012) Justicia normal neoliberal en salud: el caso de la sentencia T-760/08. Revista Convergencia Crítica: Dossié: Teoria Critica Na Atualidade, 1(2). La presente versión es una revisión aumentada y modifcada.

2 Abogado de la Universidad de los Andes. Máster en Derecho Comparado de las Universidades de Milán, Messina y Córdoba, Italia y España. Doctor en Derecho de la Universidad del Rosario.

3 Equipo investigativo del programa de salud y seguridad social de la Defensoría del Pueblo, La tutela y el derecho a la salud. Periodo 2006-2008 (Defensoría del Pueblo 2009). 
judicial, no fueron ajenos a la Corte Constitucional. En efecto, el Tribunal no se limitó a fijar pautas interpretativas sobre el derecho a la salud, sino que ordenó un conjunto de medidas para resolver los problemas de justicia con el sistema de salud, contenidas en la sentencia T-760 del 2008. En esa decisión la Corte recogió el amplio desarrollo jurisprudencial sobre el derecho a la salud, y dictó una serie de órdenes de carácter general dirigidas a superar las fallas recurrentes identificadas para la garantía del «goce universal, equitativo y efectivo» el derecho a la salud.

Uno de los aspectos más sobresalientes de esta decisión es el reconocimiento de la salud como un derecho fundamental autónomo. En este artículo nos proponemos ofrecer un análisis crítico sobre este reconocimiento que hace la Corte Constitucional, rastreando la construcción de la fundamentabilidad del derecho a la salud. Primero, presentamos un breve esquema del sistema de salud y la intervención de los jueces constitucionales, para luego analizar a profundidad la argumentación de la Corte Constitucional en la sentencia T-760/08. Retomando los planteamientos teóricos de Nancy Fraser, argumentamos que la jurisprudencia constitucional articula un discurso de «justicia normal neoliberal en salud».

\section{La organización del sistema de salud en Colombia}

Antes de la Ley 100 de 1993, en Colombia operaba el Sistema Nacional de Salud encabezado por el Ministerio de la Salud y fragmentado en tres subsistemas: (i) la seguridad social que afiliaba de forma obligatoria a los trabajadores del sector formal y la burocracia del Estado; (ii) el privado compuesto por planes de medicina prepagada según la capacidad de pago o el gasto de bolsillo para comprar los servicios requeridos; y (iii) el resto de la población o era atendida por los hospitales públicos y las entidades sin ánimo de lucro, o no podían acceder a los servicios de salud (Pinto, 2002; Yepes, Ramírez, Sánchez, Ramírez, \& Jaramillo, 2010). Los servicios de salud estaban financiados por las transferencias directas que hacía el Estado hacia los hospitales públicos, modelo conocido como subsidios a la oferta (Franco, 2002). 
En su momento, diversos estudios evidenciaron que este Sistema Nacional de Salud era altamente segmentado, con enormes inequidades e ineficiente en la utilización de los recursos disponibles (Jaramillo, 1999), lo cual motivó que a inicio de la década de los noventa se implementara en Colombia una reforma estructural al sistema de salud (Yepes et al., 2010).

Este diagnóstico del Sistema Nacional de Salud, así como la orientación de su reforma estructural reflejan el contexto de la crisis de acumulación capitalista que se registró a nivel mundial en la década de los años setenta - en donde se combinaron altas tasas de desempleo y la aceleración de la inflación, que anunciaban un periodo de estancamiento económico a nivel global- . Tendencia que fue agravada con la crisis petrolera de 1973 producto del embargo de la Organización de Países Exportadores de Petróleo (OPEP), que profundizó la caída de la paridad oro-moneda dispuesta en Bretton Woods (Harvey, 2007).

Ante el desgaste de las políticas keynesianas para hacer frente a esta crisis, las ideas de un reducido grupo de académicos, liderados por Friedrich von Hayek, fueron retomar un enfoque económico neoclásico de la mano invisible del mercado, que para el momento era minoritario, ante el auge de las políticas de intervención de Keynes en el contexto internacional. Sin embargo, son estas las premisas que van a dar paso al desmonte del Estado de Bienestar, cuando logran la influencia práctica en diversos ámbitos políticos. Esta corriente va a adquirir mayor fuerza con el otorgamiento del Premio Nobel de economía a Milton Friedman en 1976, profesor de la Universidad de Chicago, y su mentor, Hayek quien recibió el mismo premio en 1974 (Harvey, 2007).

La consolidación definitiva del neoliberalismo como ortodoxia político-económica en la dirección del Estado se dará con el ascenso al poder de Margaret Tatcher en Gran Bretaña y de Ronald Reagan en Estados Unidos en 1979. Ambas administraciones proporcionaron el apoyo político para desmontar los pactos del Estado de Bienestar entre el capital y los trabajadores para dar paso a la reducción de los impuestos que estimularan la creación de empresa, crear entornos de confianza inversionista, privatizar las empresas públicas, desregular los mercados y crear nuevos, en aquellas áreas que eran exclusivas del Estado, como la seguridad social y la atención sanitaria (Harvey, 2007). 
En esta lógica, el Estado como proveedor y centro de la salud pública y los servicios de salud, como lo era Colombia con el anterior Sistema Nacional de Salud, debe ser reemplazado por la privatización y la descentralización (World Bank, 1988), procesos que van a estar jalonados por instituciones multilaterales como el Banco Mundial y el Fondo Monetario Internacional (Useche, 2008). En la región de América Latina, las reformas (Stocker, Waitzkin, \& Iriart, 1999).

Entre 1985-1993 se desarrolló la reforma estructural al sistema de salud en Colombia. Inició con la Ley 10 de 1990 que introdujo la descentralización en el campo de la salud. Posteriormente, la Asamblea Nacional Constituyente de 1991 profundizó la descentralización y estableció la salud como servicio público cuya prestación la haría el sector privado. Este proceso finalizó con la Ley 100 de 1993, que organizó el esquema del aseguramiento en regímenes según la capacidad de pago, y las reglas del acceso a la póliza de salud. Este modelo se sustenta a partir de dos elementos: el aseguramiento y la competencia regulada.

\section{El aseguramiento}

Por mandato de la Constitución de 1991, al Estado colombiano le corresponde garantizar el acceso universal al servicio público de salud. La Ley 100/93 establece dos regímenes de aseguramiento: el contributivo y el subsidiado, que estratifican a la población de acuerdo con su capacidad de pago. Mientras se alcanza el aseguramiento universal se prevé que la población que está por fuera de estos regímenes reciba atención como "vinculados al sistema".

En el régimen contributivo se encuentran afiliados los trabajadores con contrato laboral o los independientes que tengan un ingreso de por lo menos un salario mínimo. La financiación proviene de los aportes de las personas que compran este seguro de salud sin recibir aportes del Estado. La prima que se debe pagar por este seguro corresponde al $12.5 \%$ del ingreso básico del trabajador. Quienes cuentan con un contrato laboral, el empleador aporta el $8.5 \%$ y los empleados el $4 \%$ restante, mientras que los «trabajadores independientes» deben aportar la totalidad del 12.5\%. 
El 1.5\% del aporte de los afiliados al régimen contributivo se transfiere al régimen subsidiado para ayudar a su financiamiento (Yepes et al., 2010).

En el régimen subsidiado están todas las personas sin capacidad de pago. Este régimen es producto del cambio estructural en la organización del sistema de salud que reemplaza los subsidios a la oferta, asignación presupuestal a los hospitales, por el subsidio de la demanda, sustentado en la compra de seguros de salud por parte de los diferentes gobiernos locales a las empresas aseguradoras (Gaviria, Medina, \& Mejía, 2006).

\section{La competencia regulada}

El nuevo sistema de salud puso fin al monopolio estatal del aseguramiento y separó los procesos administrativos de la prestación de los servicios de salud. Las Entidades Promotoras de Salud (EPS), en su mayoría de carácter privado, tienen a su cargo la gestión administrativa del aseguramiento. Las EPS conforman redes de servicios de salud, las cuales pueden ser propias o contratadas, conformadas por Instituciones Prestadoras de Salud (IPS), que también pueden ser de carácter público o privado (Yepes et al., 2010).

Las funciones del Estado son principalmente de regulación, dirigida a corregir las imperfecciones del mercado. Para ello, se creó la Comisión de Regulación en Salud (CRES) ${ }^{4}$, conformada por el Ministro de la Protección Social, quién la preside, el Ministro de Hacienda y Crédito Público y cinco comisionados expertos designados por el Presidente de la República.

La CRES tiene a su cargo, entre otras funciones, promover un modelo de competencia regulada a través del Plan Obligatorio de Salud (POS) que es aquel conjunto de actividades, procedimientos, intervenciones, servicios, insumos y medicamentos al que tiene derecho cada persona asegurada. El POS fue concebido como un listado cerrado y fijo de procedimientos y medicamentos, con una serie de exclusiones explícitas que en ningún caso estarían cubiertas por el seguro médico. Para financiar el POS se diseñó la

4 Esta comisión fue creada con la ley 1122/07, estas mismas funciones estaban a cargo del Consejo Nacional de Seguridad Social en Salud (CNSSS) que estaba compuesto por representantes de diversos actores del sistema, gobierno, aseguradoras, prestadores de servicios, sindicatos, empleadores, pensionados (Yepes, et al., 2010). 
Unidad de Pago por Capitación (UPC), valor que se le reconoce a las EPS por cada persona que tenga asegurada, y que debe cubrir los costos de atención del POS.

A través de la regulación, el Estado debe fomentar la competencia entre las distintas empresas aseguradoras (EPS) y redes de servicios (IPS) por la calidad y la eficiencia en las gestiones administrativas y la prestación de los servicios de salud, en donde el mercado, como gran telón de fondo, debe optimizar el uso de los recursos disponibles. Sin embargo, quince años después de la reforma y ante un aumento considerable del gasto en salud los balances sobre los resultados alcanzados son contradictorios. Por una parte, los reformistas y el gobierno coinciden en afirmar el importante avance en la cobertura de aseguramiento, el mayor acceso a la primera consulta de la población asegurada y la reducción del gasto de bolsillo.

No obstante, otros estudios señalan que estos resultados positivos deben ser analizados críticamente, por ejemplo, el avance en la cobertura del aseguramiento debe examinarse frente al contenido del POS, el cual está estratificado según la capacidad de pago. Por otra parte, estudios indican que el aseguramiento no garantiza la calidad de la atención y los servicios. También se ha reportado el deterioro en los indicadores de mortalidad evitable, en el programa de control de la tuberculosis y el incremento en la mortalidad materna y perinatal (Yepes et al., 2010).

Otros estudios evidencian las diversas barreras de acceso a los servicios de salud, ello ha sido uno de los principales motivos por los cuales la ciudadanía ha acudido a la acción de tutela, con el fin de que le sea garantizado su derecho a la salud (Abadía \& Oviedo, 2009).

\section{La intervención de los jueces constitucionales y la implementación del sistema de salud}

Desde sus primeras decisiones, la Corte Constitucional ha sostenido una postura amplia respecto a los derechos fundamentales, en contra de las posiciones tradicionales de considerar únicamente como fundamentales aquellos derechos contenidos en el capítulo I título 2 "De los derechos fundamentales" de la Constitución. Tampoco aceptó la Corte la interpretación restrictiva de la Constitución de reducir los derechos fundamentales al 
catálogo cerrado de derechos de aplicación inmediata del artículo 85 de la Constitución de 1991 (Arbelaez, 2006).

En una de las primeras decisiones de la Corte sobre el derecho a la salud, en la sentencia T-406/92 la Corte revisó la acción de tutela interpuesta por la falta de terminación del alcantarillado a cargo del Estado. La Corte consideró que el saneamiento público y la salud pueden ser derechos fundamentales por su conexidad con principios esenciales dentro de un Estado Social de Derecho tales como la vida y la dignidad humana.

Esta interpretación de la conexidad entre la salud y otros derechos fundamentales abre una línea jurisprudencial múltiples veces reiterada por la Corte; en especial, cuando aborda problemas específicos con el sistema de salud: tratamientos médicos a una persona enferma de sida, mora en la prestación de los servicios para enfermedades graves o la desprotección de grupos específicos como los ancianos o los niños, entre otros (Arbelaez, 2006).

La mayoría de conflictos de acceso al sistema de salud giran en torno al POS y los servicios que están por fuera de dicho paquete $e^{5}$. La comprensión original del POS excluía a las enfermedades ruinosas o catastróficas, a los enfermos de SIDA y otro tipo de malestares que no alcanzaba los estándares de costo-eficiencia con que se reglamentó dicho instrumento. Al respecto, la Corte indicó que esta concepción excluyente del POS no es compatible con el derecho a la salud dentro de un Estado Social de Derecho, en especial, cuando se trata de «sujetos de especial protección constitucional», para quienes la salud es un derecho fundamental. En consecuencia, la Corte aplicó la excepción de inconstitucionalidad del POS y ordenó los tratamientos, medicamentos y demás servicios requeridos.

Dado que estos servicios se encuentran por fuera del esquema de financiación (POS - UPC), la Corte definió que la carga financiera no podía ser traslada a la EPS que negó el servicio, ya que no se debía a una actuación negligente, sino una falla de todo el sistema. En consecuencia, los costos financieros de los servicios tutelados por fuera del POS debían ser

5 Equipo investigativo del programa de salud y seguridad social de la Defensoría del Pueblo, op. Cit. Nota 5. 
Justicia normal neoliberal en Salud: un análisis crítico de la sentencia T-760/08 de la Corte Constitucional

reembolsados a la EPS, confiando en la capacidad de ahorro del sistema de salud a través del Fondo de Solidaridad y Garantía, FOSYGA6́.

No obstante, el criterio de la conexidad para la tutela del derecho a la salud resultaba demasiado amplio, generando que cualquier demanda en salud fuera protegida por la Constitución. Esto incrementó las acciones de tutelas y la formación de un POS jurisprudencial, compuesto por aquellos procedimientos que de forma reiterada eran negados por las EPS y que la Corte ordenaba su prestación.

En la sentencia SU-111/97 la Corte Constitucional tecnificó su jurisprudencia y adoptó criterios precisos para determinar cuándo la salud puede ser considerada como un derecho fundamental. En dicha oportunidad la Corte redefinió la interpretación de la conexidad con un criterio más exigente, el del "mínimo vital".

De esta forma se pretendió reducir la tutela para aquellos casos en donde la pretensión era absolutamente imprescindible para la conservación de la vida, en palabras de la Corte: "El Estado social de derecho exige esforzarse en la construcción de las condiciones indispensables para asegurar a todos los habitantes del país una vida digna dentro de las posibilidades económicas que estén a su alcance. El fin de potenciar las capacidades de la persona requiere de las autoridades actuar efectivamente para mantener o mejorar el nivel de vida, el cual incluye la alimentación, la vivienda, la seguridad social y los escasos medios dinerarios para desenvolverse en sociedad" (sentencia T-426, 1992).

A partir de la sentencia SU-480/97 la Corte inició a sistematizar el conjunto de condiciones que debía verificar cada juez constitucional para que de forma legítima y moderada se justificara la intervención judicial en el sistema de salud. Para ello, la Corte estableció una serie de reglas precisas con el propósito de acceder al POS jurisprudencial sin afectar el equilibrio financiero del sistema. A su vez, el CNSSS intentó que los conflictos del sistema fueran resueltos internamente, para lo cual expidió la resolución 5061 de 1997 que reglamentaron los Comités Técnico Científicos (CTC)

6 El Fondo de Solidaridad y Garantía FOSYGA es una cuenta adscrita al Ministerio de la Protección Social manejada por encargo fiduciario, sin personería jurídica ni plante de personal propia, cuyos recursos se destinan a la inversión en salud. Artículo 218 ley 100/93; artículo 1 Decreto 1283/96. www.fosyga.gov.co 
con el objeto de "establecer las condiciones y el procedimiento para la prescripción de medicamentos no incluidos en el listado (POS), con criterios de costo-efectividad" (Arbelaez, 2006, p. 3).

Sin embargo, la introducción de los CTC no fue suficiente, y el número de acciones de tutela desde 1999 hasta el año 2006 se profundizó ${ }^{7}$. Esto genera una masificación de la jurisprudencia de la Corte a través de los "fallos de reiteración". Los jueces de tutela se limitan a citar la regla fijada por la Corte para acceder a servicios no POS y verificar el cumplimiento de dichas condiciones en el caso concreto.

En un texto que "ha llegado a ser canónico" (Arbelaez, 2006, p. 3) la Corte encuentra que se vulnera al derecho a la salud en conexidad con el mínimo vital cuando en el caso concreto se verifican las siguientes condiciones, regla que se mantiene aún vigente:

a) que la falta de tratamiento o medicamento excluido por la reglamentación legal o administrativa amenace los derechos fundamentales a la vida, el mínimo vital; b) que no exista un medicamento o tratamiento sustituto dentro del POS, o que de existir, no tenga la misma efectividad; c) que el paciente esté en incapacidad económica de sufragar el costo del medicamento o tratamiento o que no pueda acceder a dicho tratamiento por medio de otro plan o seguro médico; d) que medicamento hubiere sido prescrito por el médico tratante adscrito a la EPS a la que está afiliado el paciente.

\section{La sentencia T-760/08: el derecho a la salud como fundamental autónomo}

La Corte Constitucional a través de su Sala Segunda de Revisión de Acciones de Tutela, integrada por los magistrados Manuel José Cepeda, Jaime Córdoba Triviño y Rodrigo Escobar Gil dictó la sentencia número T-760 de 2008. La ponencia de esta sentencia estuvo a cargo del juez Manuel José Cepeda, quién para la época estaba por dejar su cargo de juez

7 El derecho a la salud en perspectiva de derechos humanos y el sistema de inspección, vigilancia y control del Estado colombiano en materia de quejas en salud (Procuraduria General de la Nación 2008). 
constitucional. La sentencia es emitida por una sala de revisión compuesta por solo tres magistrados y no por la Sala Plena de la Corte Constitucional.

Según el reglamento de la Corte Constitucional una decisión de tan amplia trascendencia y dirigida a la unificación del precedente jurisprudencial en materia de salud, debe ser adoptada por el pleno de los magistrados que componen la Corte Constitucional. Si bien, algunos análisis sobre esta decisión han resaltado el carácter democrático que caracteriza a las órdenes de la sentencia, enmarcada en una "democracia dialógica" con los otros entes del Estado involucrados, tal parece que al interior de la propia Corte Constitucional no se evidenció el mismo carácter democrático por la forma en la cual fue adoptada la decisión.

Este hecho da cuenta de las agendas políticas que se articulan al interior de la Corte Constitucional por parte de ciertos magistrados y su visión particular sobre el papel del juez constitucional, que no necesariamente responden a una concepción unitaria de todos los jueces que componen la Corte en un momento determinado. En esa medida la trayectoria del juez Cepeda no puede pasarse por alto al momento de analizar esta sentencia, quien fue el ponente y el encargado de impulsar esta decisión al interior de la sala de revisión.

Manuel José Cepeda hizo parte del kínder de Gaviria, fue quien ideó y participó en la estrategia jurídica para el cambio constitucional —Abogado de la Universidad de los Andes, con estudios de posgrado en derecho en la Universidad de Harvard-, lo ponen en contacto con la teoría jurídica estadounidense y el papel del juez constitucional en ese país. Tras dejar su cargo como Magistrado, Cepeda fungió en el gobierno Santos (2010-2014) como asesor a la reforma a la salud, a través de la ley estatutaria.

Retomando los planteamientos de la Corte Warren, el juez Cepeda implementó decisiones de amplia trascendencia a nivel nacional, entre las que se destaca la sentencia T-025 de 2004. En dicha decisión, también producto de una sala de revisión se declaró "el estado de cosas de inconstitucionalidad" en materia de desplazamiento forzado y dictó una serie de órdenes generales con el fin de superar dicha situación.

Tras esta decisión, la sala de revisión, bajo el liderazgo de Cepeda, inició un complejo proceso de seguimiento al cumplimiento de las órdenes de la sentencia. En un importante esfuerzo, con el fin de establecer si 
el indicador de "goce efectivo de derechos" se garantizaba a través de las acciones de asistencia a la población desplazada. Luego de diversos autos sobre el desarrollo de la sentencia, audiencias públicas, y sin que se diera una declaración de la superación del estado de cosas de incostitucionalidad. Tras la salida del juez Cepeda de la Corte Constitucional se conformó una sala de seguimiento especial para el cumplimiento de las órdenes de la sentencia T-025/04.

En una presentación de la sentencia T-760/08, en medio de un espacio académico, el magistrado auxiliar que participó en su elaboración, Aquiles Arrieta, anunció como una importante novedad que esta era la primera sentencia de la Corte Constitucional que incorporaba un índice, con el fin de llamar la atención sobre la extensión de la decisión. La sentencia T-760 tiene un total de 431 páginas, 35 órdenes, de las cuales 16 son de carácter general, además de dos anexos sobre los antecedentes de los casos acumulados y un recuento histórico del desarrollo de los instrumentos internacionales sobre el derecho a la salud.

El magistrado ponente decidió acumular un conjunto amplio de acciones de tutela, todas relacionadas con barreras en el acceso a los servicios de salud y en su mayoría referido a casos anteriormente resueltos de forma reiterativa por la jurisprudencia constitucional. La sentencia contiene un amplio y detallado análisis de la jurisprudencia de la Corte Constitucional sobre el derecho a la salud cuando se implementa el sistema de salud de la Ley 100 de 1993.

En esta sentencia se avanzó en dar una detallada radiografía de la vasta jurisprudencia de la Corte Constitucional sobre el derecho a la salud que da cuenta de las dificultades con el sistema de salud expuestas ante los jueces constitucionales, sin que la solución de cada caso concreto permitiera corregir las fallas de regulación que según la Corte no había sido objeto de análisis hasta dicho momento.

La acumulación de casos tenía como fin obtener una muestra representativa de los conflictos que se plantean entre los usuarios y el sistema de salud. A su vez, estos casos permiten, en criterio de la Corte, analizar las distintas "facetas" del derecho a la salud, para así valorar sus efectos, con el fin de garantizar el "goce universal, equitativo y efectivo de este derecho". 
Para su análisis, la Corte dividió los problemas jurídicos en concretos y generales. En los concretos resolvió los conflictos puntuales y avanzó en la construcción dogmática del derecho a la salud para definirlo como un derecho fundamental autónomo.

En los problemas jurídicos generales se ocupó de los elementos de regulación para la protección del derecho a la salud. En la Corte los problemas generales se resumen en la siguiente pregunta: ¿Las fallas de regulación constatadas en la presente sentencia a partir de los casos acumulados y de las pruebas practicadas por esta Sala, representan una violación a las obligaciones constitucionales que tienen las autoridades competentes de respetar, proteger y garantizar el derecho a la salud para asegurar su goce efectivo?

La respuesta de la Corte es afirmativa, por lo que dictó una serie de órdenes de alcance general con el fin de corregir las fallas de regulación.

\subsection{La salud como derecho fundamental autónomo: simplificación argumentativa}

El punto de partida para este análisis es la noción de salud. Para la Corte Constitucional es necesario superar la visión de la salud como ausencia de enfermedad. En este sentido, adopta la conceptualización de la OMS que entiende por la salud: "un estado completo de bienestar físico, mental y social dentro del nivel posible de salud para una persona". Acudiendo al bloque de constitucionalidad y los instrumentos internacionales que desarrollan este derecho precisó: "el derecho a la salud comprende el derecho al nivel más alto de salud posible dentro de cada Estado, el cual se alcanza de manera progresiva".

Advirtió que la sentencia no pretende zanjar el debate en torno a los "derechos fundamentales", no obstante, la jurisprudencia constitucional ha logrado un consenso sobre dos criterios que permiten establecer cuándo se está ante un «derecho fundamental»: "i) que existe un consenso en la dogmática constitucional sobre su fundamentabilidad y ii) que el derecho constitucional esté funcionalmente dirigido a lograr la dignidad humana y sea traducible en un derecho subjetivo".

La Sala de Revisión encontró, en el amplio precedente judicial sobre el derecho a la salud, que las anteriores dos condiciones estaban acreditadas, 
por lo que concluyó que la estrategia de la conexidad que había acuñado la jurisprudencia sobre este derecho había caído en desuso dado que, de forma cada vez más frecuente, se han reconocido situaciones especiales en donde el derecho a la salud tiene el carácter de fundamental de forma autónoma y por lo tanto, dicha estrategia para proteger el derecho constitucional a la salud resulta "artificiosa".

Se destaca que esta decisión de la Corte Constitucional abandone la doctrina de la conexidad para la protección del derecho a la salud, lo cual permite una importante simplificación en la argumentación de la persona que acude a la acción de tutela para la protección de su derecho a la salud, dado que, ya no tendrá que demostrar, en su caso concreto, que la amenaza o vulneración de su derecho a la salud presenta una conexidad verificable con otros derechos fundamentales, en especial, con el mínimo vital.

Otro aspecto que vale la pena resaltar de esta doctrina jurisprudencial es que el accionante tampoco tendrá que recurrir a la doctrina de los "sujetos de especial protección constitucional", argumentando ser parte de este grupo que ha sido definido por la jurisprudencia de la Corte para lograr que la salud sea considerada como un derecho fundamental. Actualmente, con la nueva jurisprudencia de la Corte, en principio, basta simplemente con alegar la vulneración u amenaza del derecho a la salud, ahora considerado como fundamental autónomo.

Sin embargo, asignarle el carácter de fundamental al derecho $\mathbf{\partial}$ la salud no significa que siempre que sea invocado este derecho, automáticamente se cumpla con el requisito de la "fundamentabilidad" para la procedencia de la acción de tutela, y que este reconocimiento implique que se tiene derecho a cualquier atención o servicio de salud. Al respecto, la Corte ha sido enfática en indicar que los derechos fundamentales no son derechos absolutos, por el contrario, son susceptibles de ser limitados, siempre y cuando se respeten unos límites de razonabilidad y proporcionalidad fijados por la jurisprudencia constitucional.

De lo anterior emerge la siguiente pregunta: ¿Cuál es el ámbito de protección del derecho fundamental a la salud?, es decir, ¿cuál es el contenido de este derecho y qué tipo de demandas sobre el derecho a la salud pueden ser consideradas como constitucionalmente legítimas? Al respecto, de la revisión jurisprudencial antes reseñada se evidencia que 
Justicia normal neoliberal en Salud: un análisis crítico de la sentencia T-760/08 de la Corte Constitucional

existe un amplio margen de interpretación por parte de la Corte, incluso en la sentencia T-760/08 se afirma explícitamente que: "la Sala de Revisión no entra a establecer en detalle el alcance y contenido del concepto de derecho fundamental, en general, ni con relación al caso concreto de la salud", y más adelanta explica que "el ámbito del derecho fundamental a la salud está delimitado por la dogmática constitucional, que reconoce los contenidos garantizados a las personas en virtud del mismo".

En este sentido la Corte superando la división ideológica de generaciones de derechos, explica que hablar de "derechos prestacionales" como usualmente se califican a los "derechos sociales" es un error categorial. En su concepto, todos los derechos fundamentales son prestacionales, en el sentido que demandan del Estado una serie de obligaciones de acción u omisión. Por ello adopta la visión más técnica de "facetas" de los derechos, que divide en positivas y negativas.

Estas facetas prestacionales del derecho a la salud deben cumplir con tres requisitos previos, a saber: i) la existencia efectiva de una política pública; ii) que dicha política esté orientada a garantizar el goce efectivo del derecho; iii) que contemple mecanismos de participación de los interesados. Al encontrar verificadas estas condiciones para el caso del derecho a la salud, la Corte Constitucional se abstiene de declarar que en materia de salud se está ante un estado de cosas de inconstitucionalidad, como lo hizo para el desplazamiento forzado. Este resulta ser un elemento clave sobre el real alcance de la sentencia y las posibilidades que ofrece para la defensa del derecho a la salud.

En la sentencia T-025 de 2004 la Corte Constitucional definió una regla general con el fin de establecer si existe o no un estado de cosas de inconstitucionalidad ${ }^{8}$. A primera vista, las cinco condiciones que expone la

8 "Dentro de los factores valorados por la Corte para definir si existe un estado de cosas inconstitucional, cabe destacar los siguientes: (i) la vulneración masiva y generalizada de varios derechos constitucionales que afecta a un número significativo de personas; (ii) la prolongada omisión de las autoridades en el cumplimiento de sus obligaciones para garantizar los derechos; (ii) la adopción de prácticas inconstitucionales, como la incorporación de la acción de tutela como parte del procedimiento para garantizar el derecho conculcado; (iii) la no expedición de medidas legislativas, administrativas o presupuestales necesarias para evitar la vulneración de los derechos. (iv) la existencia de un problema social cuya solución compromete la intervención de varias entidades, requiere la adopción de un conjunto complejo y coordinado de acciones y exige un nivel de recursos que de- 
Corte Constitucional son verificables de forma evidente para el caso de la salud. Pero la Corte Constitucional no solo se abstiene de una declaración de este tipo, sino que su diagnóstico sobre la situación de la salud es producto de la falta de regulación, así como de una regulación incorrecta. Una lectura política posible de esta decisión es que abstenerse de declarar un «estado de cosas de inconstitucionalidad en salud» es el apoyo que la Corte Constitucional da al modelo de la Ley 100 de 1993, que tanto en sus diversas decisiones de constitucionalidad ha considerado que la Ley 100 de 1993 y sus reformas se ajustan a la Constitución. El mensaje de la Corte fue claro: era necesario dar cabal cumplimiento a los mandatos de la Ley 100 de 1993.

La Corte analiza el bloque de constitucionalidad y el desarrollo que en el ámbito del derecho internacional de los derechos humanos ha tenido el derecho a la salud sustentado en la obligación general No. 14 del Comité de Derechos Económicos, Sociales y Culturales. En un estudio detallado de las obligaciones de i) respeto; ii) protección y iii) cumplimiento (garantizar), aunque para el tercer bloque de obligaciones la Corte puntualiza que existe un debate doctrinal sobre su contenido.

La Corte indicó que resulta constitucionalmente legítimo no incluir ciertos servicios médicos dentro del POS, dado que el derecho a la salud es limitable y la Constitución no ordena su autorización dado que: "su exclusión no desconoce aspectos importantes de la salud o de la vida del interesado". De esta forma la Corte en su jurisprudencia ha señalado que el derecho a la salud debe estar ajustado a las prioridades que los órganos competentes del sistema dispongan, con el fin de "asignar de manera eficiente los recursos escasos disponibles".

De igual manera, la Corte reseñó un amplio conjunto de decisiones judiciales anteriores que negaron la procedencia de la acción de tutela por tratarse de servicios médicos por fuera del POS que no desconocen el derecho a la salud. Al respecto, la Corte enumera los siguientes servicios de salud, constitucionalmente legítimos para ser excluidos de su garantía por el Estado: i) servicio de salud estéticos, ii) gafas y cirugías de ojos; iii)

manda un esfuerzo presupuestal adicional importante; (v) si todas las personas afectadas por el mismo problema acudieran a la acción de tutela para obtener la protección de sus derechos, se produciría una mayor congestión judicial”. 
tratamientos de fertilidad; iv) tratamiento de desintoxicación; v) prótesis; vi) bypass gástrico; vii) servicios de odontología; y viii) alergias, entre otros.

Por su parte, la Corte identificó una serie de situaciones específicas en donde el derecho a la salud adquiere la condición de fundamental autónomo y en caso de amenaza o vulneración puede ser protegido por el juez de tutela; acerca de ello, la Corte señaló los siguientes eventos:

i) Acceso a los servicios de salud que se requieran contemplados en los planes obligatorios POS; ii) Los contenidos de la ley 972/05 sobre la atención de enfermedades ruinosas o catastróficas, según el art. 2 de la citada ley; iii) Cuando no se garantiza a todos los seres humanos igualmente dignos y se establecen exclusiones discriminatorias, como el caso de parejas homosexuales cuando la pareja era excluida del régimen de salud; iv) cuando se afecte de forma grave y directa un mínimo vital, por fuera del cual el deterioro orgánico impide una vida normal.

\subsection{Consolidación de la regla para el acceso a los servicios no POS. Un cambio en la gramática constitucional}

En la sentencia la Corte Constitucional va a reiterar «la regla útil al capital» formulada para el acceso a los servicios de salud y que ya fue reseñada. Sin embargo, en la ratificación de la regla se hace una simplificación "gramatical", pero que en el fondo intenta avanzar en una interpretación estricta de la condición económica del quién acude a la acción de tutela.

Para resumir la Corte Constitucional consideró que una EPS viola el derecho a la salud cuando no autoriza un servicio de salud que no está en el POS cuando el accionante "requiera" del mismo. Para la Corte, se entiende que se "requiere" un determinado servicio de salud cuando se reúnen las siguientes características:

1. La falta del servicio médico vulnera o amenaza los derechos a la vida y a la integridad personal de quien lo requiere;

2. El servicio no puede ser sustituido por otro que se encuentre incluido en el plan obligatorio 
3. El servicio médico ha sido ordenado por un médico adscrito a la entidad encargada de garantizar la prestación del servicio a quien está solicitándolo.

En el caso concreto, además de requerir dicho servicio, se evidencia su "necesidad", lo cual se cumple, en criterio de la Corte, cuando:

El interesado no puede directamente costearlo, ni las sumas que la entidad encargada de garantizar la prestación del servicio se encuentra autorizada legalmente a cobrar, y no puede acceder al servicio por otro plan distinto que lo beneficie

La Corte Constitucional concluye que toda persona tiene derecho a acceder a los servicios de salud "que requiera", según las reglas fijadas para acceder a esos servicios por el sistema de salud. Cuando ese servicio que se "requiere" no está incluido dentro del plan de beneficios POS, la persona deberá asumir el costo adicional de dicho servicio. Sin embargo, si se logra comprobar que la persona carece de los medios económicos para acceder a los servicios, y se logra verificar esta condición, es posible autorizar los servicios que "requiere con necesidad", para que sean prestados por la EPS y a esta luego le sea reembolsado el valor de los mismos ante el Fosyga.

\section{Las órdenes generales de la T-760/08: fallas regulatorias y cumplimiento de la ley 100 de 1993}

El elemento más sobresaliente de la sentencia es el conjunto de órdenes generales que dictó la Corte Constitucional. Este ha sido el aspecto más resaltado por la dogmática constitucional como innovador en el desarrollo de los Tribunales Constitucionales, de superar los límites que impone el caso concreto estudiado por los jueces, con el fin de avanzar en una mirada más amplia y que engloba la problemática social reiterativa, para identificar las causas y atacar el problema desde la raíz, y no brindar soluciones temporales y tendientes a casos puntuales.

La Corte Constitucional realizó un diagnóstico sobre el sistema de salud con el fin de identificar las causas que están generando el incremento desproporcionado en la utilización de la acción de tutela. La Corte retomó su análisis jurisprudencial con el fin de identificar las fallas recurrentes que eran expuestas en las acciones de tutela. 
A partir de estos elementos, la Corte Constitucional consideró que las fallas estructurales del sistema de salud se debían a fallas de regulación, las cuales pretendió corregir a partir de sus órdenes generales. Dichas órdenes se enmarcan dentro del sistema de la Ley 100 y la mayoría de sus medidas están obligando a cumplir con lo dispuesto en la ley. En este sentido, la sentencia de la Corte está exigiendo que se dé cabal aplicación al sistema de salud, corrigiendo las distorsiones que han desembocado en el uso reiterativo de la acción de tutela.

Las 16 órdenes generales las agrupó la Corte Constitucional en: 1) medidas relacionadas con el plan de beneficios; 2) medidas con el derecho al recobro de servicios no POS; 3) medidas para la protección del derecho a la información en salud; carta de derechos y carta de desempeño. En este orden de ideas, la Corte Constitucional en su sentencia intenta enderezar el derecho practicado hacia los mandatos del derecho letrado y superar su separación por la vía de la regulación.

En los análisis que se han realizado sobre la sentencia se ha concluido que estas órdenes generales están enmarcadas y siguen de cerca los límites que el modelo de la Ley 100/93 y sus posteriores reformas. Para la Corte Constitucional el modelo de la Ley 100 de 1993, que ha sido analizado en detalle, debe ser considerado como una política pública en salud pues cumple con las condiciones definidas para la garantía y protección del derecho a la salud.

Por lo tanto, lo que evidenció la Corte fue la falta de cumplimiento de lo estipulado en la ley, como el conjunto de órdenes sobre el plan de beneficios para su unificación, definición puntual, abandono de las zonas grises, y la regulación precisa para que fuera a través de los procedimientos definidos al interior del sistema de salud, y no a través de la acción de tutela como se resolvieran los conflictos que plantean los usuarios.

Las medidas sobre los recobros estaban dirigidas a mejorar el flujo de los recursos en el sistema de salud, ante las reclamaciones de las EPS por las demoras, los engorrosos procedimientos para obtener los recursos que fueron utilizados para cubrir los costos de atención ordenados por los CTC o una orden judicial.

Tras la emisión de la sentencia T-760 de 2008 se institucionalizó el proceso de seguimiento a sus órdenes generales. En la sesión de la Sala Plena 
de la Corte Constitucional del 1 de abril de 2009 se decidió conformar una sala especial para esta tarea compuesta por tres magistrados, a saber: Jorge Iván Palacio Palacio, Mauricio González Cuervo, Gabriel Eduardo Mendoza Martelo.

\section{El cumplimiento de las órdenes generales de la sentencia T-760/08: la emergencia social en salud}

La propuesta de la Corte Constitucional al diálogo deliberativo para resolver la crisis en el sistema de salud, a partir de sus órdenes generales, recibió una respuesta autoritaria, vertical y de fuerza, característica del gobierno Uribe. Por coincidencia o como hecho simbólico, 16 años después de ser aprobada la Ley 100 de 1993 un 23 de diciembre, en ese mismo día, pero del año 2009 el presidente Álvaro Uribe Vélez, que ya había liderado la aprobación de la Ley 100 en el Congreso, decretó un estado de excepción haciendo uso de las facultades extraordinarias consignadas en la Constitución.

El decreto 4975 de 2009 declaró el estado de emergencia social en salud. Aunque no apareció explícito en las consideraciones del decreto para su expedición, en reiteradas oportunidades el presidente Uribe indicó en su momento que la emergencia social tenía como propósito cumplir con las órdenes generales que fueron emitidas en la sentencia T-760/08 por la Corte Constitucional.

En las consideraciones del decreto de declaratoria de la emergencia social se consignaron las razones jurídicas que motivaron la declaratoria de la emergencia social y que desarrollan a grandes rasgos las medidas que fueron adoptadas para conjurar la crisis, estas luego fueron plasmadas en 15 decretos. La razón fundamental que invoca el gobierno para la declaratoria de la emergencia social radica en la prestación de servicios no POS, esto es, el que llamamos POS jurisprudencial.

En ese sentido, la emergencia social busca responder con una "terapia de choque" como la calificó el diario inglés The Economist, con el objeto de frenar los altos costos que representa para el sistema de salud la prestación de servicios no contemplados en el plan obligatorio de salud, en una afrenta 
Justicia normal neoliberal en Salud: un análisis crítico de la sentencia T-760/08 de la Corte Constitucional

directa en contra de la labor de los jueces constitucionales en la defensa y garantía del derecho a la salud a través de su jurisprudencia.

Aunque la emergencia social en salud solo hace referencia a la Corte Constitucional y únicamente a la sentencia T-760 de 2008, toda la argumentación está dirigida al amplio desarrollo jurisprudencial de la Corte, y las decisiones de todos los jueces constitucionales del país en su jurisprudencia consolidada sobre el derecho a la salud, para que sea el ciudadano el que tenga que cubrir los costos de su atención por fuera del POS de forma individual y no tenga que ser asumido dicho valor por el Estado.

La argumentación del gobierno parte por reconocer la salud como un derecho fundamental, según lo dispuesto por la Constitución Política y la jurisprudencia de la Corte Constitucional. Sin embargo, explica que es un derecho que cuenta con limitaciones de acuerdo con los principios de eficiencia, universalidad, solidaridad, dignidad humana, así como la realidad socio-económica y la capacidad financiera del Estado.

En la argumentación fáctica para sustentar la declaratoria de la emergencia social, el gobierno presentó cuatro argumentos. El argumento principal, que tiene mayor desarrollo, es el alto costo del POS jurisprudencial; debido al mecanismo del recobro. Como no es responsabilidad de la EPS la negación del servicio no POS, sino del diseño del sistema, deben ser con estos recursos que se paguen dichos servicios. Para el caso de régimen contributivo dichos recursos son pagados con los recursos de la cuenta de solidaridad del FOSYGA, y en el régimen subsidiado dicha suma es cubierta por el ente territorial. Estas fueron las reglas establecidas por la Corte Constitucional ante el vacío regulativo de la Ley 100 de 1993, que no previó cuál sería el procedimiento y los mecanismos para la financiación de servicios de salud por fuera del paquete de beneficios (POS).

El gobierno constata un crecimiento "abrupto y acelerado" en la demanda de servicios de salud por fuera del plan obligatorio, tanto en el régimen contributivo como en el subsidiado. A su vez, ha podido verificar que algunos agentes del sistema de salud y reguladores están induciendo la oferta y la demanda de este tipo de servicios, sin tener en cuenta "(...) criterios de efectividad, sostenibilidad, costo eficiencia, racionalidad en el 
uso de los servicios y recursos, como tampoco a la propia capacidad socioeconómica de los pacientes (...)" [énfasis nuestro].

Además de ejemplificar con cifras el aumento en el número de recobros en el año 2009, se tomó el estudio que elaborara la Asociación de Laboratorios Farmacéuticos de Investigación y Desarrollo, Afidro. Según ese estudio se encontró que el recobro de medicamentos se estaba haciendo al FOSYGA por valores mayores al costo de esos medicamentos en relación con el precio de venta del laboratorio.

En otros casos el número de medicamentos recobrados superaba al número de medicamentos que el laboratorio reportaba como vendidos, y otro tipo de prácticas, así como actividades fraudulentas que venían siendo denunciadas de tiempo atrás por el observatorio del medicamento de la Federación Médica Colombiana, pero que solo fue hasta este momento en que el gobierno lo utilizaba para justificar la declaratoria de la emergencia social en salud.

Esta dinámica del sistema estaba generando un círculo vicioso de falta de recursos, en tanto que se estaría frente a una quiebra inminente de las entidades territoriales que no están pagando a las EPS, del régimen subsidiado, por los servicios prestados, lo que se replicaba en las IPS que no tenía recursos para poder seguir prestando sus servicios ante la falta de recursos económicos, al no recibir el costo de los servicios prestados que estaban por fuera del plan obligatorio en salud.

Las EPS del régimen contributivo aumentaban su cartera de deuda con las IPS, en tanto dichos servicios están por fuera de esquema de financiamiento de la UPC. Por ello, el argumento explica que, de mantenerse ese nivel de demanda de servicios del POS jurisprudencial para el primer semestre del año 2010, se acabaría con los excedentes del FOSYGA, con los que se han cubierto dichos servicios.

El segundo presupuesto fáctico para justificar la emergencia social fue el problema del flujo de los recursos, que ante las dinámicas del sistema y su "mayor complejidad" se ha evidenciado que los procedimientos establecidos para el giro, pago y distribución dispuestos en la ley son insuficientes al momento de mantener un flujo constante de los recursos entre los actores del sistema. Esto ha degenerado en un uso ineficiente, desvíos de los 
Justicia normal neoliberal en Salud: un análisis crítico de la sentencia T-760/08 de la Corte Constitucional

dineros, promoviendo un incremento en los costos financieros para el funcionamiento del sistema.

Sobre el flujo de recursos también se explicó que hacen falta mecanismos expeditos que permitan solucionar las controversias entre los actores, y tampoco se han podido incorporar al flujo recursos disponibles para el financiamiento del sistema. El tercer aspecto, es el de las barreas de acceso a los servicios de salud como consecuencia de la "territorialidad del aseguramiento". Finalmente, el cuarto elemento es que en algunos casos los recursos destinados a la salud no han tenido esa destinación específica, sino que se han desviado.

Frente a este panorama, el gobierno argumentó que se desborda la capacidad de respuesta del Estado ante esta compleja situación, por lo que las medidas administrativas, e incluso la reforma de la Ley 1122 de 2007, son insuficientes en respuesta a la superación de la crisis. También argumentó que los ingresos del Estado son exiguos, si se quiere mantener el esquema de aseguramiento alcanzado y lograr cumplir con el mandato de la universalidad y la unificación en el paquete de servicios, esto es, que exista un mismo POS para el régimen contributivo y el régimen subsidiado, como fue ordenado por la Corte Constitucional, que sin mencionarlo, se está refiriendo a una parte de las órdenes generales dictadas en la sentencia T-760/08, obligaciones que estaban contenidas en el texto de la Ley 100 de 1993 inciso segundo artículo 113.

En este orden de ideas, el gobierno derivó graves consecuencias para la protección y garantía del derecho a la salud. Explica que el POS jurisprudencial en ambos regímenes está afectando el esquema de aseguramiento, generando dificultades para mantener los niveles alcanzados y ampliar la cobertura a fin de cumplir con la meta de la universalización.

En su criterio, también se ponen en riesgo a los actores del sistema, en especial, a las Empresas Promotoras de Salud, EPS, las Instituciones Prestadoras de Salud, IPS, y las Entidades Territoriales, que sufren una grave crisis financiera y de liquidez, lo que genera dificultades para el financiamiento global del sistema,-asimismo la equidad en el acceso a los servicios de salud. Otros riesgos inminentes según el gobierno es el cierre de hospitales públicos, el cese de pago al "talento humano" en salud y hasta una parálisis en la prestación del servicio público. 
Por lo tanto, a lo largo de su argumentación y en repetidas oportunidades el gobierno explicó que se trata de una grave amenaza contra el derecho a la salud ante el deterioro de la sostenibilidad financiera del sistema de salud.

Entre las medidas urgentes a tomar para superar la crisis del sistema explicó que se apropiarían más recursos para el año 2009 y en el presupuesto del año 2010; se definirían las condiciones para la unificación del plan de beneficios POS y sus condiciones de acceso; se implementarían instrumentos que permitan avanzar en la equidad y oportunidad en el flujo de los recursos; dar "más transparencia" y eficiencia en el uso de los recursos económicos, así como corregir sus fallas, demoras y desviaciones; fortalecer los mecanismos de inspección, vigilancia y control para atacar la evasión de los recursos del sistema; conseguir nuevas fuentes de financiación al incrementar los impuestos en la explotación de los juegos de azar y ciertos bienes como los cigarrillos, el tabaco y la cerveza; fortalecimiento a la Comisión de Regulación en Salud; diseñar esquemas para la racionalización en la actuación de actores del sistema como los médicos tratantes, los usuarios, empleadores, EPS, IPS en materia de acceso a medicamentos y medidas preventivas para la recuperación de recursos, e incluso punitivas para responsabilizar a quienes incurran en conductas fraudulentas.

Frente a los servicios no POS, denominados por los decretos como "prestaciones excepcionales en salud", se propuso que las personas compren seguros como planes adicionales y complementarios o de medicina prepagada con el propósito de cubrir dichos servicios. Para el gobierno, los servicios que excedían el POS debían ser considerados como servicios suntuosos, pues el plan de beneficios contendría todos los servicios mínimos requeridos.

A su vez, se dispusieron medidas para que los trabajadores puedan acceder a sus cesantías a fin de cubrir los costos en esa situación. En caso de carecer de recursos, las personas deben someterse a un riguroso escrutinio que determine con exactitud su capacidad de pago para cubrir dichos servicios y poder acceder a fórmulas de préstamo por el sistema bancario. Solo cuando quede demostrada su incapacidad económica, se podrá ser acreedor de un subsidio en salud, en casos excepcionales y luego del estricto escrutinio de la capacidad patrimonial del paciente. 
Ante la avalancha de críticas al gobierno por estas decisiones, que demandó una defensa a ultranza no solo de las medidas, sino del sistema de salud, se suman las denuncias por corrupción en el desarrollo de los decretos, pues quienes participaron en la redacción tenían en su momento estrechas relaciones con las EPS. Entre ellos estaba el médico Augusto Galán, expresidente de ACEMI, quien, tres años atrás, fue denunciado por recibir honorarios al mismo tiempo como presidente de ACEMI y consultor del Ministerio de Salud; de igual modo participó, Teresa Tono, médico, miembro de la Junta Directiva de la EPS que representa a la compañía aseguradora Positiva.

Resultaba paradójico que ante la "crisis financiera" del sistema de salud se contratara para asesorías multimillonarias a médicos con conflicto de intereses. Otro aspecto que salió a debate fue el de los nuevos impuestos a los licores, que excluyen a licores de alta gama como el whisky y el vodka para favorecer a familiares del Ministro de Hacienda, Oscar Iván Zuluaga, dedicados a ese negocio.

A través de la red social "Facebook" se convocó a una marcha en contra de los decretos de la emergencia social. A dicha convocatoria se sumaron las diversas organizaciones sociales y colectivos que luchan por la defensa del derecho a la salud. Con una masiva participación los diversos sectores sociales manifestaron su rechazo a las medidas del gobierno. La academia también apoyó a las organizaciones sociales expresando su desaprobación con respecto a las medidas y explicando cómo se trataba de una crisis financiera para salvar el negocio de las EPS, y no una reforma estructural del sistema de salud.

Esta movilización brindó el apoyo necesario para que la Corte Constitucional en la sentencia C-252 de 2010 determinara que la declaratoria de la emergencia social era inconstitucional y por lo tanto, las medidas adoptadas con ocasión de dicha norma quedaban viciadas de inconstitucionalidad. Sin embargo, en una interpretación controvertida, mantuvo las medidas adoptadas en la emergencia de nuevos impuestos por considerarlos vitales para solventar la crisis en salud. 


\section{Posibilidades, límites y peligros de la sentencia T-760/08}

La sentencia T-760/08 ha generado un amplio debate público en el país sobre la salud como derecho fundamental y el sistema de salud para su garantía real y efectiva. No obstante, los análisis disponibles han tendido más hacia la celebración del fallo de la Corte Constitucional, y su examen crítico ha sido poco.

En este sentido, recogemos la invitación del profesor Óscar José Dueñas, quien hace un llamado a la prudencia y a no sacralizar algunas sentencias de la Corte Constitucional, como la sentencia que se analiza y el caso del desplazamiento forzado (T-025/04). Según Dueñas, este tipo de sentencias "es conveniente discutirlas, ya que incluyen temas que no se pueden aceptar a la ligera". Ambas decisiones, la T-760/08 y la T-025/04, ameritan un análisis sereno, indica el autor, dado que "admiten algunas críticas" (Ruiz-Rodriguez, Wirtz, \& Nigenda, 2009).

En primer lugar, la sentencia T-760/08 ofrece varias posibilidades sobre la discusión pública de la salud. En su momento, la sentencia reactivó el debate sobre la salud en Colombia y el modelo implementado con la Ley 100, ya que tras la expedición de la Ley 1122 de 2007 se había reducido a la implementación de la reforma y darle un compás de espera al modelo de la Ley 100 de 1993. Con la sentencia se posicionó la salud como un tema de la agenda política, ello permitió proponer nuevamente la discusión entre defensores y contradictores del sistema de salud. Esta es una posibilidad que ofrece la sentencia de abrir el debate y discutir en la agenda pública la configuración del modelo de salud para la garantía real y efectiva del derecho fundamental a la salud.

Las órdenes generales dictadas por la Corte Constitucional en cierta medida podrían ofrecer algunas alternativas con el fin de lograr la garantía del derecho a la salud, sin que los usuarios tengan que enfrentarse en una batalla legal contra las aseguradoras privadas por los servicios de salud no prestados. Además, la posibilidad de que las organizaciones sociales se lograran articular en torno al seguimiento y cumplimiento de las órdenes generales dictadas por la Corte Constitucional, que les permitiera mantener el debate sobre el sistema de salud. 
Sin embargo, como ya indicábamos, dichas órdenes estaban circunscritas con estricto apego al modelo de la Ley 100 de 1993. Ante el desconocimiento palmario de la ley, la Corte Constitucional establece en su sentencia que las autoridades encargadas del Sistema de Salud deben dedicarse a cumplir la ley. La literatura ha resaltado de forma excesiva la postura progresista de la Corte Constitucional al exigir el cumplimiento de la ley, al considerar el potencial emancipador de las decisiones de la Corte y la construcción de una contra-hegemonía al interior del Estado, de un aliado para los movimientos sociales y sus reivindicaciones. En este sentido, la sentencia queda limitada al modelo de la Ley 100 de 1993, que como hemos analizado en detalle, no está diseñado para la garantía de la salud como derecho, sino hacia el negocio de la salud y la acumulación por parte de los captadores de renta del sistema de salud.

En este sentido, las decisiones de la Corte Constitucional no atacaban las causas estructurales de la crisis de la salud en Colombia, sino que a partir de unas órdenes generales, intentaban que se diera cumplimiento a la Ley 100 de 1993, sin que lograra trascender los estrechos márgenes del sistema de salud, y avanzar hacia una construcción de un nuevo modelo de salud, ante el congelamiento político que mantiene una reforma estructural por parte del Congreso de la República y el Gobierno que seguía insistiendo que la Ley 100 de 1993 es la mejor alternativa para organizar los servicios de salud.

La Corte tampoco consideró otros elementos, que como juez constitucional tenía conocimiento. Entre estos, se encuentra el requisito que impusieron las EPS de interponer una acción de tutela a los usuarios para acceder a los servicios de salud; tampoco hizo referencia al incumplimiento de las acciones de tutela que obliga a las personas a acudir a la interposición de varios desacatos o incluso interponer una nueva tutela para obtener el cumplimiento del fallo a favor; la discriminación de la que son sujetos los usuarios que interponen una acción de tutela ante las EPS y las IPS, en donde reciben un tratamiento especial por ser pacientes "con tutela", las instituciones aplican al extremo los «itinerarios burocráticos» y hacen más engorroso todos los trámites y procedimientos para el acceso a los servicios; la selección adversa frente a las enfermedades de alto costo, enfermedades huérfanas y varias clases de pacientes que eran rechazados o tenían mayores cargas administrativas para acceder a los servicios, que para la Corte hacen 
parte de la categoría "sujetos de especial protección constitucional"; los excesivos trámites ante las EPS y la práctica del "paseo de la muerte" no eran abordados de forma directa y sistemática por el fallo de la Corte.

De hecho, se resaltó que del conjunto de órdenes de la Corte ninguna estaba dirigida a las EPS y su enfoque fue solo en la regulación y las funciones de inspección, vigilancia y control por parte del Estado.

El peligro de la sentencia T-760/08 y los análisis que la celebran es que distraen la discusión fundamental sobre la salud en Colombia en los elementos puntuales y técnicos del sistema de salud. Por ejemplo, el tamaño del POS; los mecanismos para los recobros; la estructura de los regímenes contributivo y subsidiado, etc., que hace perder de vista el sistema de salud en su conjunto, el modelo de aseguramiento, el papel de la intermediación financiera, la mercantilización de la salud, y el modelo de negocio para la captación de rentas del sistema.

La Corte Constitucional escamotea este debate central, concentrándose en los elementos técnicos del sistema, la construcción de finas argumentaciones constitucionales y doctrinarias, pero que de forma concreta no logran realizar la salud como un derecho fundamental autónomo como fue declarado por la Corte Constitucional.

Según el último estudio de la Defensoría del Pueblo sobre el uso de la acción de tutela para la defensa del derecho a la salud, en sus conclusiones señala que: "Las tutelas en salud continúan en aumento, a pesar de que el objetivo primordial de la sentencia T-760/08 era la disminución de estas acciones". Esto significa que las órdenes generales dictadas por la Corte Constitucional no están logrando el efecto buscado, y por el contrario, se está evidenciando cada vez más una mayor vulneración del derecho a la salud.

\section{La Justicia normal en salud: otra forma útil al capital}

¿Cómo interpretar la configuración del derecho a la salud desde las decisiones de los jueces constitucionales, así como la definición de su contenido y ámbito de protección? Para una interpretación que supere los márgenes de la dogmática jurídica tradicional, se utilizaran los 
Justicia normal neoliberal en Salud: un análisis crítico de la sentencia T-760/08 de la Corte Constitucional

planteamientos de Nancy Fraser acerca de las discusiones sobre justicia y la construcción del paradigma de la «justicia normal», que complementamos con un análisis marxista sobre la justicia (Fraser, 2008).

Los planteamiento de Nancy Fraser ofrecen una reflexión sobre el horizonte de la teorización política contemporánea, que para la autora está enmarcado en la «condición postsocialista», la cual se encuentra caracterizada por: i) la inexistencia de una alternativa omnicomprensiva, como lo fue en su momento el socialismo; ii) las nuevas exigencias de justicia ancladas en el reconocimiento de las diferencias y el abandono de las cuestiones sobre la redistribución; y iii) el surgimiento del neoliberalismo globalizante, que incorpora una agresiva mercantilización y un crecimiento desmesurado de las desigualdades materiales. Si bien Fraser reconoce estar inmersa en este horizonte «postsocialista», su propuesta teórica apunta a desmarcarse de ella para exponer una reflexión crítica sobre la misma.

Nancy Fraser explica que cuando se discute sobre cuestiones de justicia, como por ejemplo la erradicación de la pobreza, las diversas posiciones comparten un conjunto de supuestos subyacentes a pesar de las radicales diferencias que puedan darse entre las distintas visiones de la justicia que evoca este debate. Siguiendo con el ejemplo, la erradicación de la pobreza está regularmente enmarcada en el campo económico, especialmente en la distribución de la riqueza. Lo mismo sucede cuando se discute la discriminación de las minorías étnicas, se comparte de forma habitual que este tipo de injusticias responden al ámbito cultural, por falta de reconocimiento de las diferencias.

En los anteriores ejemplos sobre formas de injusticia, desigualdades sociales y discriminación, subyace un supuesto de teoría social compartido: las injusticias de la pobreza son un asunto de redistribución, casi siempre económica, mientras que las injusticias culturales son un problema de reconocimiento, relacionado con las identidades. También se suele aceptar, sin que haga parte del debate, quiénes son los actores que pueden hacer reivindicaciones de justicia, que por lo general son individuales, y el tipo de instituciones a las que se deben dirigir, generalmente los Estados territoriales. Estos presupuestos compartidos sobre el marco en que se deben desenvolver las reivindicaciones de justicia constituyen en su conjunto el punto de partida de la discusión y en esa medida, no hacen parte del debate sobre la justicia. 
A pesar de la dificultad de encontrar discusiones que compartan todos los presupuestos descritos, Fraser argumenta que al estar por fuera del debate y tener algún grado de aceptación, permiten la construcción de un paradigma de «justicia normal», en donde la justicia se discute desde los presupuestos dados, sin salirse de dichos márgenes. En esa medida y retomando los planteamientos de Kuhn de ciencia normal, para Fraser, es posible considerar que también la justicia es normal: "siempre y cuando las discrepancias públicas o la desobediencia respecto a sus supuestos constitutivos se mantengan bajo control".

El conjunto de principios subyacentes en la discusión sobre la justicia admite construir una "gramática discernible" que permita establecer el paradigma de la «justicia normal». Cualquier desviación de dicha gramática será relevada a la esfera privada o será tachada como «anomalías» que no tienen lugar. Esta expulsión del marco de discusión pretende evitar la acumulación de anormalidades y desviaciones que puedan conducir a una posible desestructuración del discurso. En consecuencia, la gramática de la «justicia normal» permite reconocer cuándo se está ante reclamaciones legítimas de justicia, dado que responden al marco normalizador definido.

Las primeras decisiones de la Corte Constitucional privilegiaron una interpretación orientada hacia al carácter de «fundamental» del derecho a la salud, para lo cual elaboró diversas construcciones dogmáticas, en especial la doctrina de la «conexidad». En principio, esta interpretación de la Corte puede ser considerada como «anormal», dado que el contexto está ordenado por una gramática distinta, que considera la salud como un derecho aspiracional, de carácter prestacional que debe ser desarrollado por el Legislador. A su vez, se sostiene que la prestación de los servicios de salud involucra importantes recursos públicos que no permiten su aplicación inmediata y por lo tanto, no puede ser considerado como fundamental.

También en estas primeras decisiones la Corte sostuvo una postura crítica ante la implementación de las políticas (pos)neoliberales en salud, por ejemplo, sobre la definición del POS. En criterio de la Corte las consideraciones de costo-eficiencia con que se formula el conjunto de servicios de la póliza resultaba incompatible con el derecho constitucional a la salud, dado que la exclusión de ciertos servicios podía afectar de forma grave derechos fundamentales como la vida o la integridad personal, como sucedió en el caso de las personas con VIH-SIDA. Bajo esta interpretación, 
desde un criterio amplio de conexidad del derecho a la salud como la dignidad humana, la vida, la integridad personal en el marco del Estado Social de Derecho, la Corte logró desarrollar una justicia «anormal» que cuestionara los presupuestos del sistema de salud y a su vez fuera crítica de las políticas «neoliberales», reducidas a un examen de costo-eficiencia.

Ante el incremento de acciones de tutela sobre el derecho a la salud y las presiones por el creciente activismo de la Corte, la «anormalidad» de concebir la salud como un derecho fundamental por conexidad con criterios amplios, se va a ir reduciendo paulatinamente, para terminar normalizando la justicia en salud de forma funcional al modelo (pos)neoliberal de la Ley 100 de 1993.

La justicia normal neoliberal en salud va a consolidarse en la sentencia T-760/08, al sistematizar sus presupuestos y su forma de funcionamiento. Según esta decisión existen dos consensos de dogmática constitucional sobre el ámbito del derecho fundamental a la salud: i) cuando de la aplicación de la regulación en salud se deriva un derecho subjetivo; ii) cuando es necesaria la excepción de inconstitucionalidad ante la vulneración o amenaza del derecho a la salud.

El primer supuesto, para considerar la salud como derecho fundamental, parte de la normativa que rige el derecho a la salud, esto es, la Constitución, el bloque de constitucionalidad, las leyes y demás normas de inferior jerarquía que desarrollen este derecho, siempre y cuando sea posible concretar una garantía subjetiva. Por ejemplo, las normas que rigen el POS, establecen una garantía subjetiva, dado que este paquete de servicios debe ser garantizado a toda persona asegurada, según el régimen al que corresponda. La normalización opera al dar por sentada la reglamentación del derecho a la salud, por lo que las reclamaciones deben ajustarse a las reglas del sistema, siguiendo en el ejemplo, su demanda debe ser de algún medicamento, servicio o intervención incluida en el POS.

A su vez, la justicia de la Corte se alinea con el discurso (pos) neoliberal en la medida en que no cuestiona la lógica del aseguramiento y su circuito financiero, sino que por el contrario, encuentra que en este caso el derecho a la salud es fundamental, dado que cuando interviene en este tipo de casos encuentra que sus medidas no afectan el equilibrio financiero 
del sistema, lo que permite que la "fundamentabilidad" de la salud sea fácilmente reconocible.

El segundo consenso es que pueden existir servicios que están excluidos por la normatividad que desarrolla el derecho a la salud, pero que son requeridos con necesidad por comprometer gravemente diversos bienes jurídicos protegidos constitucionalmente como la salud, la dignidad humana o la integridad personal. Este segundo supuesto permite un espacio para una justicia «anormal» que cuestione sus presupuestos, como a su vez oponerse a las lógicas del orden (pos)neoliberal de la salud. Pero, por el contrario, en este caso también se evidencia un reforzamiento por la normalidad y el (pos)neoliberalismo.

El acceso a los servicios excluidos del POS está sometido a un estricto escrutinio y control, ejercido por los diversos actores del sistema. Se empieza por el personal médico que debe realizar su ejercicio profesional en diagnósticos a partir de la medicina basada en la evidencia, esto es, el contenido del POS, concebido como un catálogo cerrado y rígido; a su vez las EPS tienden a desincentivar los diagnósticos por fuera del POS, y establecen diversas estrategias para que los servicios médicos se mantengan dentro de dicho marco; en caso de que un médico ordene procedimientos por fuera del POS, se debe pasar a la evaluación del CTC, que con criterios de costo-eficiencia, debe establecer si la EPS autoriza el tratamiento no POS. La ley 1438 de 2011 profundizó en los procedimientos burocráticos para el acceso a los servicios de salud, que además de reformar las funciones de los CTC, introdujo las Juntas Técnico Científicas (JTC), a las cuales el Ministerio de Salud asigna las especialidades tecnológicas competentes. En caso de estar en desacuerdo con la decisión del CTC y la JTC se podrá recurrir ante la Superintendencia Nacional de Salud (SNS) para la revisión de la decisión.

Si la negación persiste, se puede acudir a la acción de tutela, previo el agotamiento del anterior trámite administrativo, y el juez constitucional debe realizar un estricto escrutinio de las condiciones que ha definido la Corte Constitucional para acceder a medicamentos o tratamientos excluidos del POS: amenaza o vulneración de derechos fundamentales como la vida, la integridad personal o el mínimo vital; que no exista otro tratamiento dentro del POS con la "misma efectividad"; que la persona no esté en capacidad económica de comprar el medicamento o acceder a 
él mediante otro esquema de aseguramiento (medicina prepagada o plan complementario); que el médico tratante se encuentre adscrito a la EPS a la que está afiliado el paciente; y constatar que los servicios de salud no estén expresamente excluidos del POS.

Después de este riguroso examen el juez constitucional puede conceder el amparo constitucional y además, ordenar que el costo de los servicios prestados sea recobrado ante el FOSYGA. Estos constituyen los requisitos para que una persona pueda acceder a servicios de salud que se encuentren excluidos del POS, complejos trámites burocráticos tanto al interior del sistema de salud como de la rama judicial, por el que deben pasar las personas para acceder a este tipo de servicios.

Esto se traduce en la normalilzación de la justicia en salud, al establecer cuáles son los parámetros que deben reunir las demandas por servicios de salud para ser considerada como constitucionalmente legítima. Estos parámetros, tanto los del sistema de salud como los judiciales no hacen parte del debate, y se erigen como los presupuestos desde los cuales se parte para hacer uso de la acción de tutela para la protección del derecho a la salud.

Por su parte, el activismo judicial de la Corte busca no alterar el equilibrio financiero del sistema, exime a las EPS de cubrir los costos de servicios por fuera del modelo de financiación POS - UPC. Esto garantiza la integralidad, brindando seguridad jurídica que permite el cálculo capitalista que requieren las EPS para implementar diversas estrategias de contención de costos, por lo que la Corte incentiva la acumulación de capital de las EPS.

A partir del consenso de la Ley 100 de 1993 y el derecho útil al capital, tanto letrado como practicado, quedan configurados los presupuestos de la «justicia normal neoliberal» en salud, en la medida en que las reivindicaciones de la salud, cuando se acude a la acción de tutela, deben estar enmarcadas en este contexto, y responder a estos parámetros, ya que las desviaciones van a ser consideradas como «anormales» y por fuera del ámbito de protección del derecho fundamental a la salud. La justicia de la Corte Constitucional en materia de salud termina colonizada por el discurso (pos)neoliberal, garantizando los derechos de propiedad privada de las EPS que bajo las fórmulas contractuales están blindadas para 
no interferir en la acumulación de capital, que la Corte permite con las órdenes de sus sentencias.

De lo anterior, se concluye que el reconocimiento de la salud como derecho fundamental que ha sido considerado como un importante avance en la protección de este derecho, tiene un alcance reducido, dado que su contenido y ámbito de protección se limita a: i) las políticas neoliberales en salud a partir de la Ley 100/93 y sus desarrollos normativos, orientados por los principios de costo/eficiencia; y ii) a ciertas excepciones que están sometidas a un estricto y riguroso escrutinio caso por caso, en donde solo ciertos servicios de salud están legítimamente protegidos por la Constitución, siempre y cuando no se afecte el equilibrio financiero del sistema y no haga parte de las exclusiones explícitas del POS.

De lo expuesto argumentamos que la definición del derecho a la salud como fundamental, configura un discurso de «justicia normal neoliberal en salud». Las reivindicaciones del derecho a la salud deben someterse a este marco normalizador para que sean protegidas por la acción de tutela, mientras que esta justicia garantiza la acumulación de capital a los entes privados que intervienen en el mercado de la salud.

\section{Referencias}

Abadía, C. E., \& Oviedo, D. G. (2009). Bureaucratic Itineraries in Colombia. A theoretical and methodological tool to assess managed-care health care systems. Social Science E Medicine, 68(6), 1153-1160.

Arango, R. (2008). El derecho a la salud en la jurisprudencia constitucional. En Alexei, J., Teoría constitucional y políticas públicas: bases críticas para una discusión. Bogotá: Universidad Externado de Colombia.

Arbeláez, R. M. (2006). La protección constitucional del derecho a la salud: la jurisprudencia de la Corte Constitucional colombiana. Derecho y Salud, 14(2), 205-240.

Botero, M. C. (2006). La acción de tutela en el ordenamiento constitucional colombiano. Bogotá: Consejo Superior de la Judicatura.

Defensoría del Pueblo. (2009). La tutela y el derecho a la salud. Periodo 2006-2008. Bogotá: Defensoría del Pueblo. 
Franco, A. S. (2002). La salud pública hoy: enfoques y dilemascontemporáneos en salud pública. Bogotá, Universidad Nacional de Colombia.

Gaviria, A., Medina, C., \& Mejía, C. (2006). Evaluating The Impact Of Health Care Reform In Colombia: From Theory To Practice (No. 002647). Documentos CEDE. Universidad de los Andes - CEDE. Recuperado de https://ideas.repec.org/p/col/000089/002647.html Harvey, D. (2007). Breve historia del neoliberalismo. Madrid: Akal S.A. Jaramillo, I. (1999). El futuro de la salud en Colombia: ley 100 de 1993 cinco años después. Santa Fe de Bogotá: Fundación Friedrich Ebert de Colombia - Fescol - Fundación Corona, Fundación para la Educación Superior -FES-, Fundación Antonio Restrepo Barco.

Pinto, M. D. M. (2002). La competencia regulada y la atención desde la perspectiva del usuario. Evidencia del sistema de salud colombiano. Mimeo, Boston: Harvard School of Public Health.

Ruiz-Rodríguez, M., Wirtz, V. J., \& Nigenda, G. (2009). Organizational elements of health service related to a reduction in maternal mortality: The cases of Chile and Colombia. Health Policy, 90(2-3), 149-155. https://doi.org/10.1016/j.healthpol.2008.09.006

Stocker, K., Waitzkin, H., \& Iriart, C. (1999). The Exportation of Managed Care to Latin America. N Engl J Med 340,(14), 1131-1136. https:// doi.org/10.1056/NEJM199904083401425

Uribe, G. M. (2009). La dinámica de los actores en la implementación de la reforma del sistema de salud en Colombia: exclusiones y resistencias (1995-2003). Revista de_Gerencia y Políticas de la Salud, 8(17), 44-68.

Useche, A. B. (2008). De la salud pública a la salud privada: una perspectiva global sobre la reforma al Sistema de Salud en Colombia. Revista Palimpsestus, 6, 123-131.

World Bank (1988). Financing health services in developing countries: an agenda for reform (English). A World Bank policy study. Washington DC; World Bank. Recuperado de http://documents.worldbank.org/curated/en/468091468137379607/Financing-health-services-in-developing-countries-an-agenda-for-reform 
Yepes, J. F., Ramírez, M., Sánchez, L.H., Ramírez, M.L., \& Jaramillo (2010). Luces y sombras de la reforma de la salud en Colombia. Ley 100 de 1993. Bogotá: Mayol Ediciones. 
